\title{
Factors associated with vaccination status among children age 12-23 months in Ethiopia, based on 2016 EDHS: logit based multinomial logistic regression analysis
}

Ermias Bekele Enyew ( $\nabla$ ermiashi@gmail.com )

Mettu University, Ethiopia

Abiyu Abadi Tareke

West Armachiho District Health Office

\section{Research Article}

Keywords: vaccination, children, EDHS, Ethiopia

Posted Date: June 11th, 2021

DOI: https://doi.org/10.21203/rs.3.rs-605017/v1

License: (c) (1) This work is licensed under a Creative Commons Attribution 4.0 International License.

Read Full License

Version of Record: A version of this preprint was published at PLOS ONE on February 25th, 2022. See the published version at https://doi.org/10.1371/journal.pone.0264004. 


\section{Abstract}

Background: Childhood immunization is one of the most cost-effective prevention measures for children's mortality and morbidity, saving 2-3 million lives per year. Therefore, the aim of this study was to identify factors associated with vaccination status among children 12-23 months of age in Ethiopia.

Method: A secondary data analysis was done based on the 2016 Ethiopian Demographic and Health Survey (EDHS). A total weighted sample of 1911 children age 12-23 months of age were included in the study. Logit based Multinomial logistic regression analysis was computed to distinguish factors associated with routine vaccination of children aged 12-23 months. P-value less than 0.05 was used to declare statistical significance of each independent variables, and adjusted Relative risk ratio (RRR) with 95\% confidence interval were used to present the result and STATA 14 was utilized for data management and analysis.

Result: Overall the prevalence of full vaccinated children was $35 \%$, while $49 \%$ of children were partially vaccinated and $16 \%$ were non-vaccinated. In multinomial analysis, having focused ANC (at least four visits) contrasted to no ANC visits at all had 9.7 higher relative risk of being fully vaccinated than not vaccinated $[\mathrm{RRR}=9.74,95 \% \mathrm{Cl}=3.52-26.94]$, and 5 times higher relative risk of being partially vaccinated than not vaccinated [RRR=4.97, 95\% $\mathrm{Cl}=2.00-12.33]$.

Conclusion: The present study found that childhood full vaccination status was low compared with the World Health Organization targets. Frequency of ANC visit and visited by field worker were significantly associated both partially and full vaccination whereas, visited health facility last 12 months and wealth status were significantly associated with childhood full vaccination.

\section{Introduction}

Childhood immunization is one of the most cost-effective prevention measures for children's mortality and morbidity, saving 2-3 million lives per year(1). Globally, over the last five years, the percentage of children receiving prescribed vaccines has remained constant. In 2019, approximately 85 percent (116 million) of infants received three doses of DTP-3/Penta-3 vaccine, and 125 countries achieved at least $90 \%$ DTP3 vaccination coverage, but approximately 19.7 million children under the age of one year have not received basic vaccines(2).

In low- and middle-income nations, childhood vaccination provides good protection against vaccinepreventable diseases $(3,4)$. While countries in Sub-Saharan Africa give high attention to childhood vaccination, child mortality related with vaccine-preventable diseases remains high and hard to reach (remote areas) of developing countries, only $50 \%$ of children and one in twenty had access to childhood vaccination(5).

In Ethiopian, EPI program was launched in 1980 with a longer-term goal of achieving $90 \%$ DPT3 coverage in all regions through strategies of reaching every district (RED) and sustainable outreach service (SOS) 
approaches and protect nearly 3-million annual births against VPDs (6). However, a large number of children have not been immunized(7). Ethiopia has exceptionally poor immunization coverage, approximately 1 million children reported to be unvaccinated and vaccine-preventable diseases accounting for about 16 percent of under-five mortality(8). Despite the progress made in lowering underfive mortality rates, about 190,000 children die each year(9).

According to the 2016 EDHS report, childhood vaccination was low in the emerging regions of Ethiopia(10). Studies have shown that maternal education, socioeconomic status, antenatal care, place of delivery, sex of child, postnatal care, media exposure, perceptions of vaccination, place of residence and cold chain management also influence childhood vaccination status $(7,11-14)$.

Different research conducted in Ethiopia on childhood vaccination have focused on either vaccination coverage of individual vaccine or complete and incomplete, vaccination and associated factor using two categories full vaccination or non -vaccination. Even if the previous studies give important clues to policymaking and other stockholder, they mainly lack of consistency and representative to be used for national level policymaking and programming by concerned bodies, as far as my literature searching, studies separated the vaccination status into non-vaccinated, partially vaccinated and full vaccinated and assorted factors among children age 12-23 month in Ethiopia are limited.

Using logit based multinomial logistic regression analysis assessing the factors that contribute to vaccination status is important in order to devise evidence-based strategies/polices that would raise the overall immunization coverage which in turn reduce the infant and child mortality. Therefore, the aim of this study was to identify factors associated with vaccination status among children 12-23 months of age in Ethiopia by using logit based multinomial logistic regression analysis based on the 2016 Ethiopian Demographic and Health Survey (EDHS).

\section{Method And Material}

\section{Study design and sampling procedures}

The study was conducted in Ethiopia. Ethiopia is located in the Horn of Africa. The current population of Ethiopia is $116,870,377$ in 2021 , with $78.3 \%$ living in rural areas based on World meter elaboration of the latest United Nations data. It has nine Regional states (A far, Amhara, Benishangul-Gumuz, Gambella, Harari, Oromia, Somali, Southern Nations, Nationalities, and People's Region (SNNP) and Tigray) and two city Administrative (Addis Ababa and Dire-Dawa). Ethiopia has followed 3 tiers of preventive healthcare system approaches. These are primary-level healthcare comprising of a primary hospital, health center, and health post; secondary-level healthcare (general hospital); and tertiary-level healthcare (specialized hospital).

A secondary data analysis was done based on the 2016 Ethiopian Demographic and Health Survey (EDHS). In 2016 EDHS, a two stage stratified sampling technique was employed to select representative samples for the country as whole. The regions in the country were stratified into urban and rural areas. 
Then, samples of enumeration areas (EAs) were selected in each stratum in two stages. In the first stage, 645 EAs were selected with probability proportional to the EA size. The EA size is the number of residential households in the EA as determined in the 2007 Ethiopian Population and Housing Census. In the second stage, a fixed number of $\mathbf{2 8}$ households per cluster were selected randomly from the household listing(10). All women aged 15-49 years who were usual members of the selected households were eligible for female survey. Children of age 12-23 months with missing age of child and outcome variable were excluded from the study. A total weighted sample of 1903 children age 12-23 months of age were included in the study.

\section{Study variables}

\section{Outcome variables}

Vaccination status: Number of children aged 12-23 months received one dose of BCG vaccine, three doses of polio vaccine, and three doses of pentavalent vaccine (DTP-hepB-Hib), three dose of pneumococcal conjugate vaccine (PCV), two dose of virus vaccine and one dose of measles vaccine was considered as "fully vaccinated"; partially vaccinated status was defined as having received some but not all vaccines; and non-vaccinated status was defined as not having received any vaccines. Fully vaccination definition is adopted from Ethiopian national HMIS (health management information system) indicators guideline (15). But, the vaccine IPV (injectable polio vaccine) was not collected in 2016 EDHS and not included in this analysis.

\section{Independent variables}

The independent variables included in this study were: maternal age, women educational status, husband/partner's education status, place of residence, women working status, wealth status, media exposure, frequency of ANC visit, region, and visited health facility last 12 months, visited by field worker, child sex, place of delivery and participation of decision-making.

\section{Operational definition:}

Participation on decision making: women who decided their health issue alone are labeled as "yes" and coded as " 1 ", who decide jointly with their partner were labeled as "some" and coded as " 2 ", while those responded their partner alone decide on their health issue were labeled as "no " and coded as " 0 ".

\section{Statistical Analysis}

Logit based Multinomial (polytomous) logistic regression analysis was computed to distinguish factors associated with routine vaccination of children aged 12-23 months. Multinomial Logistic Regression is a simple continuation of binomial logistic regression model to be used when the dependent variable is nominal and has more than two categories. Children who were not vaccinated to any routine vaccination were considered as referent category and specified as " 0 " and the remaining categories i.e. fully vaccinated and partially vaccinated were considered as alternative category (not the reference category). 
Variance inflation factors (VIF) were assessed to check multicollinearity among the variables. A VIF value greater than 10 was considered as an indication of multi-collinearity; however, no significant multicollinearity was observed. P-value less than 0.05 was used to declare statistical significance of each independent variables, and adjusted Relative risk ratio (RRR) with $95 \%$ confidence interval were used to present the result. The parameter RRR indicates the likely to membership of one category of the independent variable compared reference category. The closer a value of RRR to zero the less effect of the explanatory variable has on the dependent variable's alternative category as compared to reference category.(16)

\section{Ethical approval and consent to participate}

Authors have requested DHS Program through an online request by written letter of objective and significance of the study. Permission for data access was granted to download and use the data from http://www.dhsprogram.com. The EDHS programs permitted data access, and data were used for only the current study.

\section{Result}

A total of 1,911 (weighted) children aged 12 to 23 months were included in this study. The majority of the children $(11.1 \%)$ are at the age of one year. The children's and mother's mean age were 16.9 months and 29.18 years, respectively.

Generally, in Ethiopia prevalence of full vaccinated children was $35 \%$, while $49 \%$ of children were partially vaccinated and $16 \%$ were non-vaccinated. In terms of maternal age fully vaccinated children were exhibited among women aged $15-19(40.6 \%)$ and $20-29(38.4 \%)$ years. Conversely, partially vaccinated children are highly noticed among women aged 40-49 (56.4\%) \& 30-39 (49.3\%) years. Regarding educational status of maternity of uneducated women $624(52.5 \%)$ and $329(28 \%)$ their children belongs to partially vaccinated and fully vaccinated respectively. Of 713 women who give birth at health facility to their last birth, more than half of their children were partially vaccinated. (Table 1) 
Table 1

distribution of vaccination status of children aged 12-23 months by selected characteristics 2016 EDHS.

Characteristics

Vaccination stath

$15-19$

20-29

30-39

40-49

Child sex

Male

Female

Maternal educational status

no education

232(19.7\%)

$66(11.8 \%)$

Primary

Secondary

Higher

Occupation

Working

Not working

Place of delivery

Home

Health facility

Frequency of ANC visit

No ANC

1_3 visit

$>=4$ visits

$7(6.6 \%)$

$4(5.5 \%)$

$110(12.7 \%)$

198(19.2\%)

413(47.2\%)

522(50.2\%)

$349(40.1 \%)$

$318(30.6 \%)$

873

1038

329(27.8\%)

227(40.8\%)

63(61.2\%)

48(73.4\%)

66

1185

558

102 


\begin{tabular}{|c|c|c|c|c|}
\hline \multirow{2}{*}{$\begin{array}{l}\text { Characteristics } \\
\text { Yes }\end{array}$} & \multicolumn{3}{|c|}{ Vaccination status } & \multirow{2}{*}{$\begin{array}{l}\text { total } \\
942\end{array}$} \\
\hline & $112(12.0 \%)$ & $398(42.0 \%)$ & $432(46.0 \%)$ & \\
\hline \multicolumn{5}{|c|}{ Visited by field worker in the last 12 months } \\
\hline Not visited & 255(13.3\%) & $671(35.1 \%)$ & $388(20.3 \%)$ & 1314 \\
\hline Visited & $54(2.8 \%)$ & $264(13.8 \%)$ & $596(14.5 \%)$ & 596 \\
\hline \multicolumn{5}{|l|}{ Wealth status } \\
\hline Poorest & $120(24.9 \%)$ & $264(54.8 \%)$ & $97(20.3 \%)$ & 481 \\
\hline Poorer & $66(17.8 \%)$ & $185(49.1 \%)$ & 123(33.1\%) & 373 \\
\hline Middle & $60(14.2 \%)$ & $227(53.9 \%)$ & 134(31.9\%) & 421 \\
\hline Richer & $39(11.2 \%)$ & $169(47.6 \%)$ & $145(41.2 \%)$ & 353 \\
\hline Richest & $24(8.6 \%)$ & $91(32.3 \%)$ & $167(59.2 \%)$ & 282 \\
\hline \multicolumn{5}{|c|}{ Place of residency } \\
\hline Rural & $300(17.9 \%)$ & $850(50.7 \%)$ & $525(31.4 \%)$ & 1674 \\
\hline Urban & $9(3.8 \%)$ & $80(34.9 \%)$ & $140(61.3 \%)$ & 228 \\
\hline \multicolumn{5}{|l|}{ Region } \\
\hline Urban admin & $1(1.6 \%)$ & $14(21.4 \%)$ & $51(77.1 \%)$ & 66 \\
\hline Agrarian & $283(16.5 \%)$ & $857(49.5 \%)$ & $585(34.0 \%)$ & 1725 \\
\hline Pastoralist & $65(20.3 \%)$ & $65(54.0 \%)$ & $30(25.7 \%)$ & 119 \\
\hline \multicolumn{5}{|l|}{ Media exposure } \\
\hline No & $245(12.7 \%)$ & $665(47.2 \%)$ & $349(40.1 \%)$ & 1260 \\
\hline Yes & $63(19.2 \%)$ & $270(50.2 \%)$ & $316(30.6 \%)$ & 650 \\
\hline \multicolumn{5}{|c|}{ Participation on decision making } \\
\hline No & $66(18.4 \%)$ & 194(54.1\%) & $98(27.5 \%)$ & 358 \\
\hline Some & 198(16.4\%) & $563(46.3 \%)$ & $451(37.3 \%)$ & 1212 \\
\hline Yes & $25(10.7 \%)$ & $123(51.5 \%)$ & $89(37.8 \%)$ & 237 \\
\hline \multicolumn{5}{|c|}{ Husband/partner's educational status } \\
\hline None & 165(19.6\%) & $441(52.3 \%)$ & $236(28.1 \%)$ & 842 \\
\hline Primary & $98(13.7 \%)$ & $353(49.1 \%)$ & $268(37.2 \%)$ & 718 \\
\hline Secondary & $16(11.5 \%)$ & $52(36.2 \%)$ & $75(52.3 \%)$ & 1438 \\
\hline
\end{tabular}




\begin{tabular}{|c|c|c|c|}
\hline Characteristics & Vaccination status & & total \\
\hline Higher & $29(29.9 \%)$ & $59(61.0 \%)$ & 96 \\
\hline
\end{tabular}

Highest fully vaccinated children was observed in Addis Ababa, Dire Dawa, Tigray and Benishangul regions, while lowest prevalence were noticed in Somalia, Afar and Oromia region (Fig. 1).

\section{Logit based multinomial logistic regression analysis}

Output of Logit based logistic regression analysis shown in (Table 2) using the category not vaccinated as base outcome (reference group) adjusted relative risk ratio (RRR) was estimated for child being partially vaccinated along with not vaccinated and fully vaccinated along with not vaccinated. The variables associated with partially and fully vaccination were; ANC visits, visited by field worker in the last 12 months, visit to health facility in the last 12 months, place of residency and wealth status. Being visited by field workers and ANC visits were variables that predicts both fully and partially vaccination status uniformly. But, the variable place of residency predicts only partially vaccinated, while visits to HF within the last 12 months and wealth status predicts only fully vaccination.

In contrast to children from rural residents, children from urban area had 3.8 times higher relative risk of being partially vaccinated compared to not vaccinated children [RRR $=3.82,95 \% \mathrm{CL}: 1.32-11.12$ ]. In the same way, Children whose mothers visited a health facility in the past 12 months in comparison to those who did not have any visit had 2.16 greater relative risk of being fully vaccinated relative to nonvaccinated children $(\mathrm{RRR}=2.16,95 \% \mathrm{Cl}=1.4-3.52]$. 
Table 2

Association between independent variables and vaccination status among children age 12-23 months after adjusting for covariates (reference group = not vaccinated) using multivariable multinomial logistic regression.

\begin{tabular}{|c|c|c|c|c|c|c|}
\hline \multirow[t]{3}{*}{ Characteristics } & \multicolumn{3}{|c|}{ Partially vaccinated } & \multicolumn{3}{|l|}{ full vaccinated } \\
\hline & \multicolumn{3}{|c|}{$95 \% \mathrm{Cl}$} & \multicolumn{3}{|c|}{$95 \% \mathrm{Cl}$} \\
\hline & Adjusted RRR & LL & UL & Adjusted RRR & LL & UL \\
\hline \multicolumn{7}{|l|}{ Maternal age } \\
\hline $15-19$ & 1.00 & & & 1.00 & & \\
\hline $20-29$ & 1.28 & 0.36 & 4.52 & 0.75 & 0.23 & 2.47 \\
\hline $30-39$ & 0.96 & 0.26 & 3.62 & 0.48 & 0.13 & 1.85 \\
\hline $40-49$ & 1.56 & 0.32 & 7.56 & 0.82 & 0.17 & 4.03 \\
\hline \multicolumn{7}{|l|}{ Sex of child } \\
\hline Male & 1.00 & & & 1.00 & & \\
\hline Female & 0.94 & 0.60 & 1.47 & 1.05 & 0.63 & 1.75 \\
\hline \multicolumn{7}{|l|}{ Working status } \\
\hline Working & 1.00 & & & 1.00 & & \\
\hline Not working & 0.84 & 0.50 & 1.40 & 0.77 & 0.46 & 1.31 \\
\hline \multicolumn{7}{|l|}{ Place of delivery } \\
\hline Home & 1.00 & & & 1.00 & & \\
\hline Facility & 0.61 & 0.30 & 1.25 & 0.83 & 0.39 & 1.76 \\
\hline \multicolumn{7}{|l|}{ ANC visits } \\
\hline None & 1.00 & & & 1.00 & & \\
\hline 1 to 3 visits & 2.25 & $1.25^{\star \star}$ & $4.05^{\star \star}$ & 3.94 & $2.05^{\star \star \star}$ & $7.56 * * *$ \\
\hline$\geq 4$ visits & 4.97 & $2.00 * *$ & $12.33^{* *}$ & 9.74 & $3.52 * \star \star$ & $26.94 * * \star$ \\
\hline \multicolumn{7}{|l|}{ Visit to HF } \\
\hline No & 1.00 & & & 1.00 & & \\
\hline Yes & 1.18 & 0.69 & 2.01 & 2.16 & 1.32 ** & $3.52 * \star$ \\
\hline \multicolumn{7}{|c|}{ Place of residency } \\
\hline Rural & 1.00 & & & 1.00 & & \\
\hline
\end{tabular}




\begin{tabular}{|c|c|c|c|c|c|c|}
\hline \multirow{3}{*}{$\begin{array}{l}\text { Characteristics } \\
\text { Urban }\end{array}$} & \multicolumn{3}{|c|}{ Partially vaccinated } & \multicolumn{3}{|c|}{ full vaccinated } \\
\hline & \multicolumn{3}{|c|}{$95 \% \mathrm{Cl}$} & \multicolumn{3}{|c|}{$95 \% \mathrm{Cl}$} \\
\hline & 3.82 & $1.32^{*}$ & $11.12^{*}$ & 2.87 & 0.81 & 10.20 \\
\hline \multicolumn{7}{|l|}{ Region } \\
\hline urban admin & 1.00 & & & 1.00 & & \\
\hline Agrarians & 0.38 & 0.07 & 2.09 & 0.19 & 0.03 & 1.11 \\
\hline Pastoralists & 0.36 & 0.06 & 2.11 & 0.19 & 0.03 & 1.17 \\
\hline \multicolumn{7}{|c|}{ Visited by field worker } \\
\hline No & 1.00 & & & 1.00 & & \\
\hline Visited & 2.13 & $1.13^{*}$ & $4.02 *$ & 2.97 & $1.58 * *$ & $5.60 * *$ \\
\hline \multicolumn{7}{|l|}{ media exposure } \\
\hline No & 1.00 & & & 1.00 & & \\
\hline Yes & 1.05 & 0.51 & 2.18 & 1.35 & 0.69 & 2.64 \\
\hline \multicolumn{7}{|c|}{ Decision making autonomy } \\
\hline No & 1.00 & & & 1.00 & & \\
\hline Some & 0.61 & 0.34 & 1.10 & 0.83 & 0.41 & 1.71 \\
\hline Yes & 1.17 & 0.53 & 2.56 & 1.32 & 0.53 & 3.29 \\
\hline \multicolumn{7}{|c|}{ Husband's/partner's education } \\
\hline None & 1.00 & & & 1.00 & & \\
\hline Primary & 1.20 & 0.66 & 2.19 & 1.21 & 0.66 & 2.20 \\
\hline Secondary & 0.67 & 0.22 & 2.04 & 0.65 & 0.19 & 2.18 \\
\hline Higher & 0.94 & 0.15 & 5.93 & 0.52 & 0.09 & 3.11 \\
\hline \multicolumn{7}{|c|}{ Maternal education } \\
\hline None & 1.00 & & & 1.00 & & \\
\hline Primary & 1.64 & 0.77 & 3.47 & 1.77 & 0.82 & 3.80 \\
\hline Secondary & 0.65 & 0.13 & 3.18 & 1.13 & 0.25 & 5.05 \\
\hline Higher & 0.23 & 0.02 & 2.17 & 0.76 & 0.09 & 6.46 \\
\hline \multicolumn{7}{|l|}{ Wealth status } \\
\hline Poorest & 1.00 & & & 1.00 & & \\
\hline
\end{tabular}




\begin{tabular}{|lllllll|}
\hline \multirow{2}{*}{ Characteristics } & \multicolumn{2}{l}{ Partially vaccinated } & \multicolumn{5}{c|}{ full vaccinated } \\
\cline { 2 - 7 } & \multicolumn{5}{c|}{$95 \% \mathrm{Cl}$} & $95 \% \mathrm{Cl}$ \\
\hline Poorer & 1.30 & 0.63 & 2.66 & 1.89 & 0.88 & 4.03 \\
\hline Middle & 1.97 & 0.91 & 4.26 & $2.31^{*}$ & $1.07^{*}$ & $5.01^{*}$ \\
\hline Richer & 1.63 & 0.80 & 3.32 & $2.59 *$ & $1.22^{*}$ & $5.49^{*}$ \\
\hline Richest & 0.74 & 0.24 & 2.25 & 1.69 & 0.50 & 5.79 \\
\hline Constants & 4.06 & 0.40 & 41.17 & 1.86 & 0.19 & 18.53 \\
\hline $\begin{array}{l}\text { P-value*<0.05, **<0.01, ***=<0.001 } \\
\text { HF; health facility, LL; lower limit, UL; upper limit }\end{array}$ & & & & \\
\hline
\end{tabular}

Children belongs to middle wealth households relative to poorest had 2.3 higher risk of being fully vaccinated in contrast to not vaccinated children [RRR $=2.31,95 \% \mathrm{Cl}=1.07-5.01$ ]. In the same manner, children from richer households relative to poorest had 2.6 higher relative risk of being fully vaccinated than not vaccinated. $[\mathrm{RRR}=2.59,95 \% \mathrm{Cl}=1.22-5.49]$.

Children whose mothers made 1-3 ANC visits compared to no ANC visit had nearly four times higher relative risk of being fully vaccinated compared to non-vaccinated [RRR $=3.94,95 \% \mathrm{Cl}=2.05-7.56]$, and 2.2 folds higher relative risk of being partially vaccinated compared to non-vaccinated [RRR $=2.25,95 \%$ $\mathrm{Cl}=1.25-4.05$ ]. in similar fashion, having focused ANC (at least four visits) contrasted to no ANC visits at all had 9.7 higher relative risk of being fully vaccinated than not vaccinated [RRR $=9.74,95 \% \mathrm{Cl}=3.52-$ 26.94], and 5 times higher relative risk of being partially vaccinated than not vaccinated [RRR $=4.97,95 \%$ $\mathrm{Cl}=2.00-12.33]$. The relative risk of being fully vaccinated compared to not vaccinated is threefold higher in children whose mother was visited by field workers in the past 12 months compared to not visited [RRR $=2.97,95 \% \mathrm{Cl}=1.58-5.60]$, and 2.13 fold higher relative risk of being partially vaccinated in contrary to not vaccinated [RRR $=2.13,95 \% \mathrm{Cl}=1.13-4.02]$ holding other covariates constant.

\section{Discussion}

We assessed the routine vaccination coverage and risk factors for vaccination status among children aged 12-23 months in the Ethiopia using evidence from fourth Ethiopian demographic health survey. To declare as child fully vaccinated the context of Ethiopia was applied in this study. Infanthood vaccination has its own contribution to public health like reducing vaccine preventable diseases. Among the vaccine preventable diseases that can be minimized by routine vaccination; Diphtheria, hepatitis $B$, influenza, pertussis, pneumonia, tetanus, rotavirus, tuberculosis and measles (17).

This study examined predicting factors of vaccination status of children age 12-23 months using Logit based multinomial logistic regression analysis. Factors affecting vaccination status was identified 
separated for partial vaccination and full vaccination status taking not vaccinated children as reference category. From Table 1 nearly half (49\%) of the children were partially vaccinated and more than two-third (33\%) were fully vaccinated. Among predictor variables identified from this study being visited by field workers and ANC visits were common predictor variables to both alternative categories. But, visit to health facility in the past 12 months, place of residency and wealth status were not consistent (common) predictors of alternative categories.

The current study found that children born from mothers who had at least four ANC visits are 10 folds more likely to be fully vaccinated than not vaccinated compared to no ANC visits, and had five times higher relative risk of partially vaccinated than not vaccinated children compared to who had no visits. This finding is consistent with research done in Ethiopia (18), Bangladesh(19), Afghanistan(20) and Senegal (13). The same pattern of association was observed among women who had one to three antenatal care visits. A possible explanation for these results may be due to; women during the time of ANC visits they have greater chance of communicating with their healthcare providers about the importance of childhood vaccination. It is better to make additional effort to increase antenatal care visits by Policy makers, health educators and other public health concerned body.

Another crucial finding from this research was visits by fieldworker, children from visited households by a fieldworker in the past 12 months are 2.13 higher relative risk of partially vaccinated than not vaccinated as compared to not visited groups, and 3 times more risky of fully vaccinated than not vaccinated in contrary to children from households not visited by field worker in the past 12 months. Similarly, women who had visited to health facility in the past 12 months are more likely to get their children fully vaccinated than not vaccinated children in comparison to children whose mother hadn't visited. This finding broadly supported by the work of other studies in this area(19). There are several possible explanations for this result; mothers who had visited by fieldworkers are more likely to get health information related to vaccination and to discuss about vaccination status of under five children in that specific visited household. Unvaccinated/partially vaccinated children are more likely to be linked to nearby health facilities either to complete or to start up their routine vaccination schedule by the fieldworker.

Regarding place of residency compared to children inhabiting in rural areas, urban inhabitant children 3.8 times higher relative risk of partially vaccinated than not vaccinated to routine immunizations. Women from urban area are more liable to health information and good awareness about health of their children in general and vaccination status of their children in specific. Additionally, urban dweller women are more likely to get vaccinated their children due to availability and accessibility of health care system in contrast to rural dweller mothers. There is documented evidence about urban-rural difference in utilization of maternal health services in prior EDHS studies(21).

Finally, wealth status of the household had significant influence on vaccination status of children. Children from middle and richer household were more likely to be fully vaccinated. From this study, as the level of wealth increases the relative risk of being fully vaccinated than not vaccinated increases 
accordingly. However, being children to the category of richest household was not statistical significant, this may be due to small observations. These results seems in line with previous studies $(20,22)$. These relationships may partly be explained by; Women in higher territory of wealth are likely to empowered and less likely to confront challenge of transportation fee to take their child to vaccination site.

These findings may help us to plan highly focused and specific interventional initiatives based on factors predicting vaccination status of children separately i.e. for partially vaccinated, for fully vaccinated and not vaccinated. Even though, this study used national representative dataset and advanced statistical model to predict factors associated with vaccination status of children it is not free from limitations. The main limitation of this study is; unable to include predictor variables that are not collected by EDHS like knowledge, attitude toward vaccination, maternal TT vaccination. And due to cross-sectional nature of study design it is difficult to build causation types of relationships.

\section{Conclusion}

The present study found that childhood full vaccination status was low compared with the World Health Organization targets. After controlling for potential confounder, the logit based multinomial logistic regression result showed that frequency of ANC visit and visited by field worker were significantly associated both partially and full vaccination whereas, visited health facility last 12 months and wealth status were significantly associated with childhood full vaccination. As a result, enhancing ANC visit, visited by field worker and visited health facility last 12 months can help to improve childhood vaccination.

\section{Abbreviations}

RRR; relative risk ratio, ANC; Antenatal care, DPT; Diphtheria, Pertussis and Tetanus, EA; Enumeration Area, EDHS; Ethiopia Demographic and Health Survey, Cl; confidence Interval and EPI; Expanded program of immunization.

\section{Declarations}

\section{Acknowledgments}

we would like to thank the Ethiopian Central Statistics Agency for providing us with all the relevant secondary data used in this study. Finally, we would like to thank all who directly or indirectly supported us.

\section{Competing Interest}

The authors declare no competing interests. 


\section{References}

1. Organization WH. The global vaccine action plan 2011-2020: review and lessons learned: strategic advisory group of experts on immunization. 2019.

2. World Healh Organization. Immunization coverage Geneva15 July 2020 [4 may 2021]. Available from: https://www.who.int/news-room/fact-sheets/detail/immunization-coverage.

3. Lee EHJ, Lewis RF, Makumbi I, Kekitiinwa A, Ediamu TD, Bazibu M, et al. Haemophilus influenzae type $b$ conjugate vaccine is highly effective in the Ugandan routine immunization program: a casecontrol study. Tropical Medicine \& International Health. 2008;13(4):495-502.

4. Danielsson N, Fakakovikaetau T, Szegedi E. Improved immunization practices reduce childhood hepatitis B infection in Tonga. Vaccine. 2009;27(33):4462-7.

5. Wolfson LJ, Gasse F, Lee-Martin S-P, Lydon P, Magan A, Tibouti A, et al. Estimating the costs of achieving the WHO-UNICEF Global Immunization Vision and Strategy, 2006-2015. Bulletin of the World Health organization. 2008;86:27-39.

6. FMOH E. ETHIOPIA NATIONAL EXPANDED PROGRAMME ON IMMUNIZATION COMPREHENSIVE MULTI-YEAR PLAN 2016-2020. FMOH; 2015.

7. Lakew Y, Bekele A, Biadgilign S. Factors influencing full immunization coverage among 12-23 months of age children in Ethiopia: evidence from the national demographic and health survey in 2011. BMC public health. 2015;15(1):1-8.

8. Organization WH, Research SPf, Diseases TiT, Diseases WHODoCoNT, Epidemic WHO, Alert P. Dengue: guidelines for diagnosis, treatment, prevention and control: World Health Organization; 2009.

9. Health FDRoEMo. Health sector transformation plan (2015/16-2019/20). Federal Ministry of Health Addis Ababa, Ethiopia; 2015.

10. Csa I. Central statistical agency (CSA)[Ethiopia] and ICF. Ethiopia demographic and health survey, Addis Ababa, Ethiopia and Calverton, Maryland, USA. 2016.

11. Asfaw AG, Koye DN, Demssie AF, Zeleke EG, Gelaw YA. Determinants of default to fully completion of immunization among children aged 12 to 23 months in south Ethiopia: unmatched case-control study. Pan African Medical Journal. 2016;23(1).

12. Abadura SA, Lerebo WT, Kulkarni U, Mekonnen ZA. Individual and community level determinants of childhood full immunization in Ethiopia: a multilevel analysis. BMC public health. 2015;15(1):1-10.

13. Mbengue MAS, Sarr M, Faye A, Badiane O, Camara FBN, Mboup S, et al. Determinants of complete immunization among senegalese children aged 12-23 months: evidence from the demographic and health survey. BMC public health. 2017;17(1):1-9.

14. Zaidi SMA, Khowaja S, Kumar Dharma V, Khan AJ, Chandir S. Coverage, timeliness, and determinants of immunization completion in Pakistan: evidence from the Demographic and Health Survey (2006-07). Human vaccines \& immunotherapeutics. 2014;10(6):1712-20.

15. federal minister of health. HMIS INDICATOR REFERENCE GUIDE2017. 
16. Starkweather J, Moske AK. Multinomial logistic regression. 2011.

17. world health organization. vaccination and immunization 2021 [updated 30 December 2020; cited 2021 april 26]. Available from: https://www.who.int/news-room/q-a-detail/vaccines-andimmunization-what-is-vaccination?adgroupsurvey= \{adgroupsurvey\}\&gclid=CjOKCQjwppSEBhCGARIsANIs4p6z46fi5-MSTjW5bYzp0fTyAbxcw1IGtVPzBp6IC-SuGEauSLhFuYaAucREALw_wcB.

18. Tefera YA, Wagner AL, Mekonen EB, Carlson BF, Boulton ML. Predictors and barriers to full vaccination among children in Ethiopia. Vaccines. 2018;6(2):22.

19. Rahman M, Obaida-Nasrin S. Factors affecting acceptance of complete immunization coverage of children under five years in rural Bangladesh. Salud pública de méxico. 2010;52:134-40.

20. Aalemi AK, Shahpar K, Mubarak MY. Factors influencing vaccination coverage among children age 12-23 months in Afghanistan: Analysis of the 2015 Demographic and Health Survey. PloS one. 2020;15(8):e0236955.

21. Memirie ST, Verguet S, Norheim OF, Levin C, Johansson KA. Inequalities in utilization of maternal and child health services in Ethiopia: the role of primary health care. BMC health services research. 2016;16(1):1-8.

22. Porth JM, Wagner AL, Moyer CA, Mutua MK, Boulton ML. Women's empowerment and child vaccination in Kenya: the modifying role of wealth. American Journal of Preventive Medicine. 2021;60(1):S87-S97.

\section{Figures}


\begin{tabular}{ll}
\hline & $82 \%$
\end{tabular}
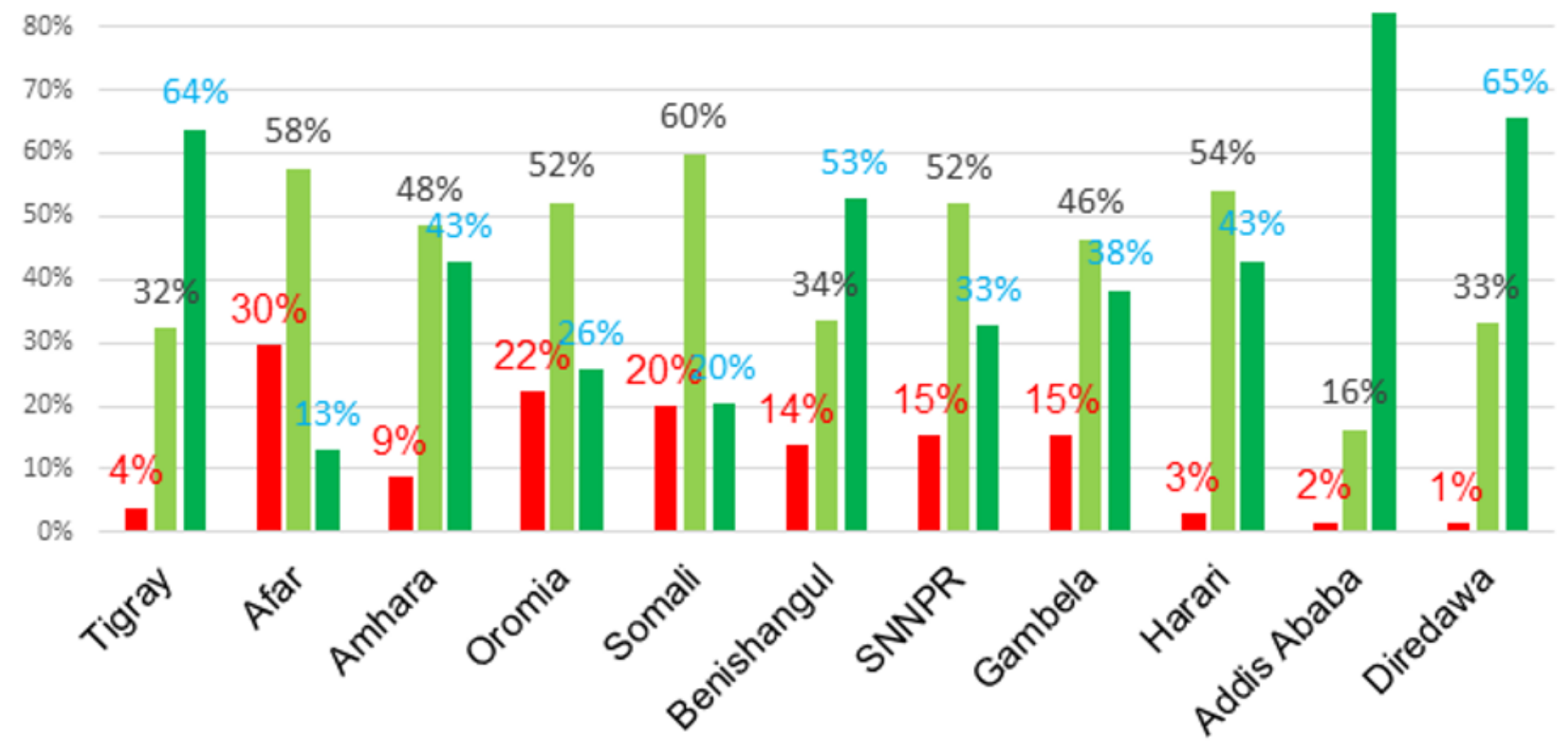

- not vaccinated $\quad$ partially vaccinated $\quad$ fully vaccinated

Figure 1

prevalence of vaccination status among 12-23 months aged children by administrative region in Ethiopia, 2016 EDHS 